\title{
Design of Vibration Sensor Based on Fiber Bragg Grating
}

\author{
Zhengyi ZHANG ${ }^{*}$ and Chuntong LIU \\ Department Two, Rocket Force University of Engineering, Xi'an, 710025, China \\ "Corresponding author: Zhengyi ZHANGＥ-mail: 18809231139@163.com
}

\begin{abstract}
Fiber grating is a kind of new type of fiber optic light source device which has been rapidly changing in the refractive index of the core in recent years. Especially, it can realize the high precision of the external parameters by means of the special structure design and the encapsulation technology $[1,2]$. In this paper, a fiber grating vibration sensor which is suitable for vibration monitoring in key areas is designed based on the technical background of vibration monitoring system. The sensor uses a single beam structure and pastes the fiber Bragg grating (FBG) to measure the vibration wavelength on the surface. When the vibration is simply harmonic vibration, the Bragg reflection wavelength will change periodically, and the periodic variation of the wavelength curve can be measured by the fiber grating demodulator, then the correctness of the experimental results is verified. In this paper, through the analysis of the data measured by the demodulator, the MATLAB software is used to verify the data, and the different frequency domains, the modes, and the phase frequency curves are obtained. The measurement range is $0 \mathrm{~Hz}-100 \mathrm{~Hz}$, and the natural frequency is $90.6 \mathrm{~Hz}$.
\end{abstract}

Keywords: Vibration monitoring; fiber Bragg grating; vibration sensor; modal analysis; frequency domain

Citation: Zhengyi ZHANG and Chuntong LIU, "Design of Vibration Sensor Based on Fiber Bragg Grating," Photonic Sensors, 2017, 7(4): $345-349$.

\section{Introduction}

Fiber Bragg grating (FBG) sensing technology is a new and multi-disciplinary cross application technology. The monitoring of vibration sensor based on the sensor technology has become an important research direction. Compared with traditional sensors, fiber grating sensor has the advantages of small volume, light weight, implantable structure, multiplexing, and no electromagnetic interference [3, 4]. The fiber grating technology developed rapidly in recent years, and it is a new type of fiber optic light source device whose core refractive index changes cyclically, especially the use of grating wavelength encoding, the structure design, and the special packaging technology can achieve absolute measurements of various external parameters with high accuracy. In this paper, based on the vibration monitoring system of fiber Bragg grating vibration sensor, a fiber grating vibration sensor is designed, which is suitable for the intrusion detection [5].

\section{Basic characteristics of fiber Bragg grating}

When $\Lambda$ and $n_{\text {eff }}$ under the influence of the external environment (temperature, stress, and pressure) $\Delta n_{\text {eff }}$ and $\Delta \Lambda$ change, it results in Bragg conditions of the wavelength shift $\Delta \lambda$ as follows:

$$
\Delta \lambda=2 \Delta n_{\text {eff }} \Lambda+2 n_{\text {eff }} \Delta \Lambda
$$

It is shown that the reflection wavelength shift is

Received: 23 March 2017 / Revised: 28 May 2017

(C) The Author(s) 2017. This article is published with open access at Springerlink.com

DOI: $10.1007 / \mathrm{s} 13320-017-0416-2$

Article type: Regular 
related to the effective refractive index of the fiber core and the variation of the grating constant. When the fiber grating is subjected to axial stress or temperature changes, $n_{\text {eff }}$ and $\Lambda$ will change [6]. Under the action of the stress, the refractive index changes, and the deformation causes the grating period to change. The photothermal effect caused by temperature changes the effective refractive index. By elastic mechanics, the central wavelength of FBG $\Delta \lambda$ is selected, and the temperature and the axial stress can be expressed as follows:

$$
\begin{aligned}
\Delta \lambda= & \lambda_{B}\left\{1-\left(\frac{n_{\text {eff }}^{2}}{2}\right)\left[P_{12}-\mu\left(P_{11}+P_{12}\right)\right]\right\} \varepsilon_{\partial}+ \\
& \lambda_{B}(\alpha+\xi) \Delta T .
\end{aligned}
$$

As the axial strain, $\varepsilon_{\partial}$ is the light, $P_{11}$ and $P_{12}$ are the elastic coefficients of fiber materials, $\mu$ is the fiber material for mu Poisson ratio, $\alpha$ is a fiber thermal expansion coefficient, $\xi$ is the thermal coefficient for zeta, and $\Delta T$ is the temperature variation [7].

\section{Principle of fiber Bragg grating vibration sensor}

From the strain sensor model of the FBG, it can be seen that if the strain of the FBG is a periodic dynamic strain that changes over time and the change is due to the vibration of the object connected to it, then the model can be used to measure the period sexual vibration (such as simple harmonic vibration) [8,9]. For a deterministic vibration, the main parameters are: acceleration, amplitude, velocity, and vibration frequency. If the acceleration and vibration frequency are known, the amplitude and velocity can be expressed by the acceleration. Therefore, the acceleration is used to express the vibration. In this way, the FBG vibration acceleration sensing system can be designed, and the basic principle is as follows: the external acceleration can be transformed into dynamic strain if the dynamic strain amplitude does not exceed the fiber grating allowed to bear the strain $\mu \varepsilon$, the Bragg wavelength changes due to the dynamic strain on the FBG, then the acceleration can be measured by detecting the change in the Bragg wavelength [10,11].

\section{Optimization of single girder type structure and physical production}

The initial model is that the length $L$ is equal to $50 \mathrm{~mm}$, and the cylinder diameter of the bottom surface $D$ is equal to $1 \mathrm{~mm}$. The model is imported into the ANSYS software to generate the entity. Using the meshing method in ANSYS, the model is meshed, as shown in Fig. 1. The fixed constraint is set on the fixed end of the model, and then the finite element modal analysis is carried out. The modal analysis in ANSYS to get the vibration cloud chart is shown in Fig. 2.

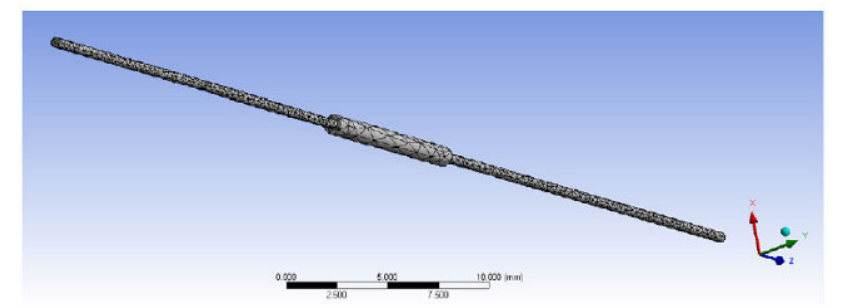

Fig. 1 Meshing the physical model.

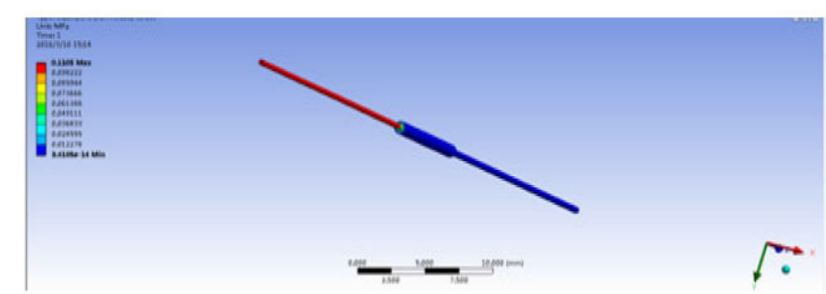

Fig. 2 Vibration nephogram obtained by modal analysis.

The first order natural frequency is $90.06 \mathrm{~Hz}$ as shown in Fig. 3.

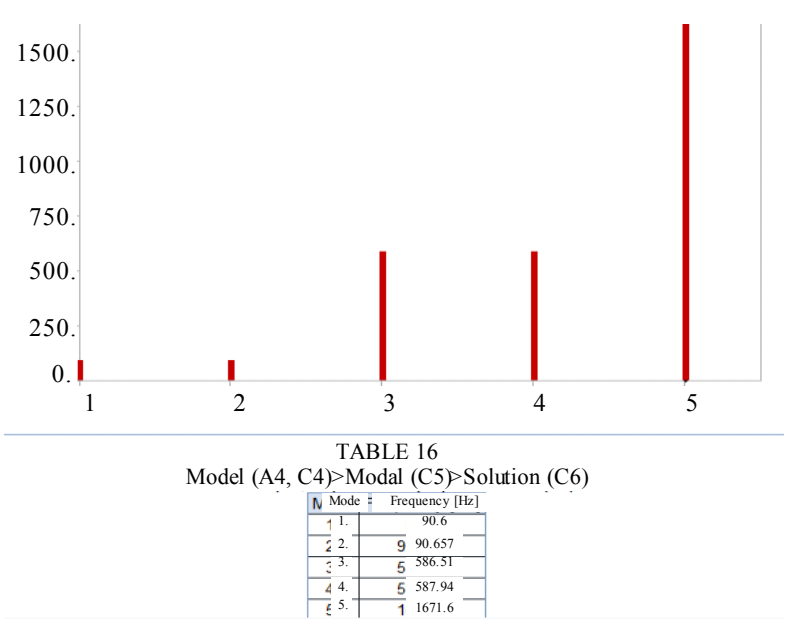

Fig. 3 Natural frequency of modal analysis. 


\section{Experimental verification}

The sensor connector is connected directly to the demodulator. The scanning mode is set to continuous scanning, and the frequency is $100 \mathrm{~Hz}$. The value of wave crest is recorded separately. The measured waveform is obtained, and the central wavelength is $1549.960 \mathrm{~nm}$ as shown in Fig. 4. After editing the MATLAB software to run the program, the time domain map is generated for data validation. When the vibration frequency of the vibration table is $33.5 \mathrm{~Hz}$, the data are obtained by MATLAB, and the frequency domain image is shown in Fig. 5.

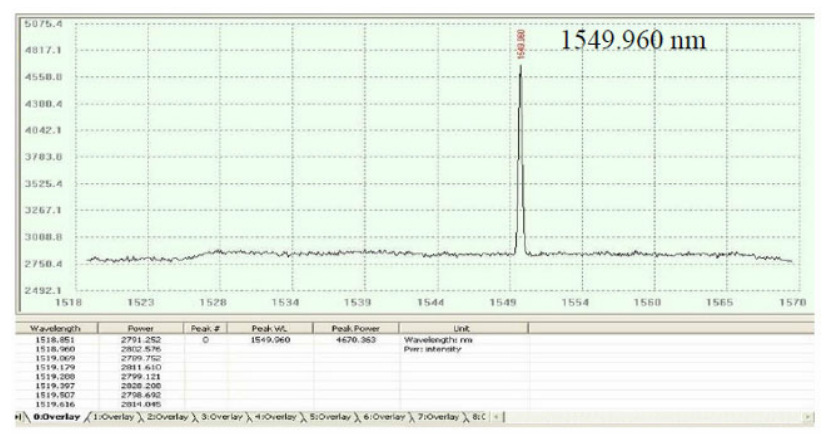

Fig. 4 Central wavelength of FBG is measured by the demodulator.

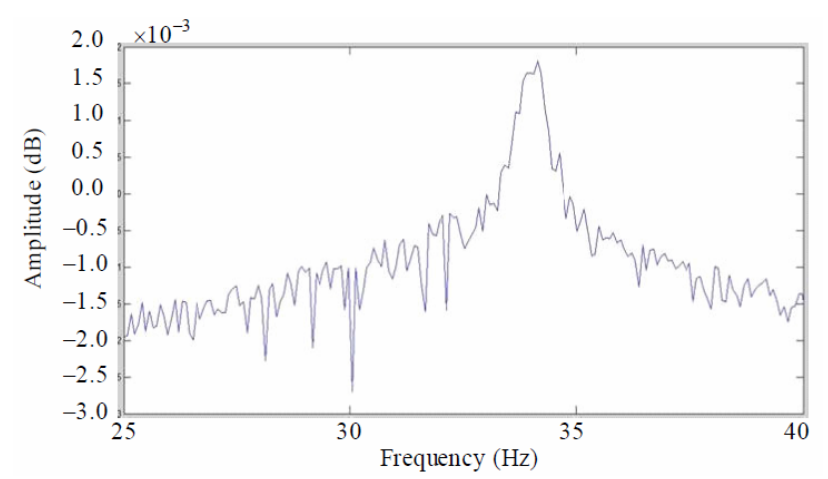

Fig. 5 Frequency domain image when the vibration frequency is $33.5 \mathrm{~Hz}$.

When the vibration frequency is $35 \mathrm{~Hz}$, the data are run by MATLAB to get the frequency domain image as shown in Fig. 6, and the time domain image is shown in Fig. 7.

According to the experimental data and the analysis results, it can be seen that the FBG central wavelength has obvious peak value, and it is close to the frequency of vibration source under vibration excitation, which indicates that the FBG vibration sensor can measure the excitation frequency of vibration source.

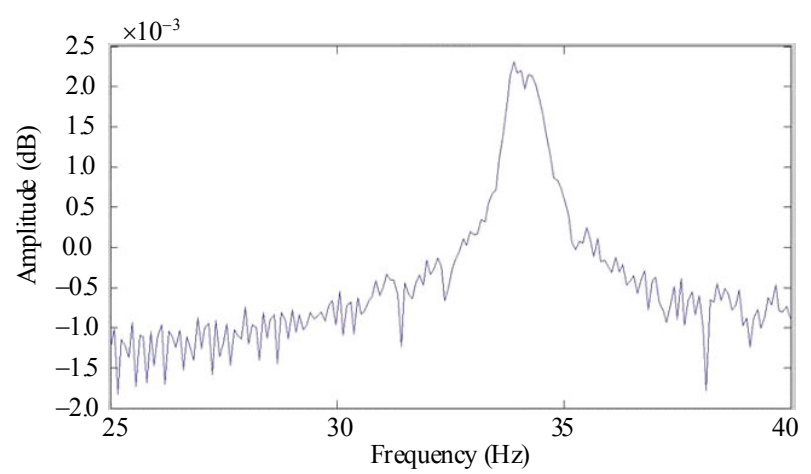

Fig. 6 Frequency domain image when the vibration frequency is $35 \mathrm{~Hz}$.

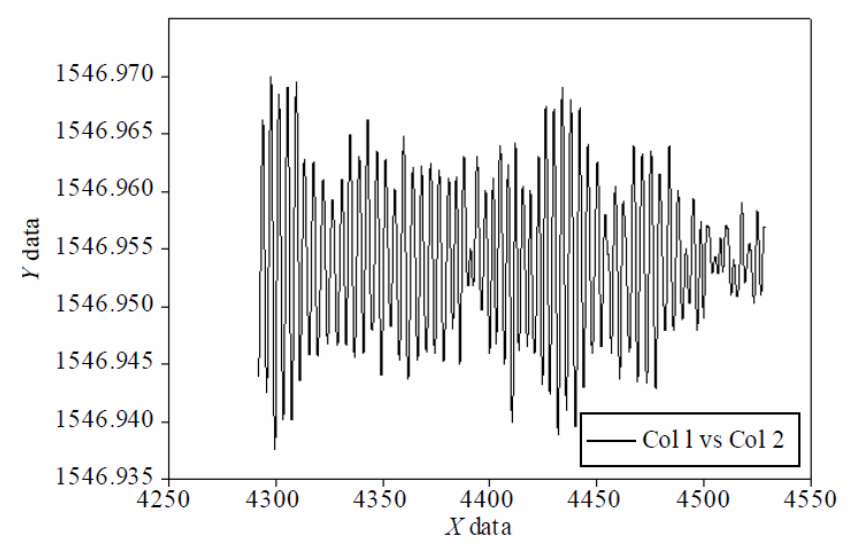

Fig. 7 Frequency domain of $35 \mathrm{~Hz}$.

\section{Optimization structure of the measurement data analysis}

In the experiment, the sensor is sensitive to different forms of vibration signals, and different characteristics of the waveform are given. From Fig. 8, we can read the corresponding changes in the central wavelength and vibration frequency, and from frequency domain images, we can also see two peaks appear at the 14-Hz low frequency. The first peak is not the experimental table set frequency, and it is caused by the resonance between shaking table and experimental table vibration of the vibration table caused by the table as shown in Fig. 8 . Because when the vibration table is set too high, the vibration frequency value will have a large jitter, so we choose a lower vibration frequency as shown in Fig. 9. 


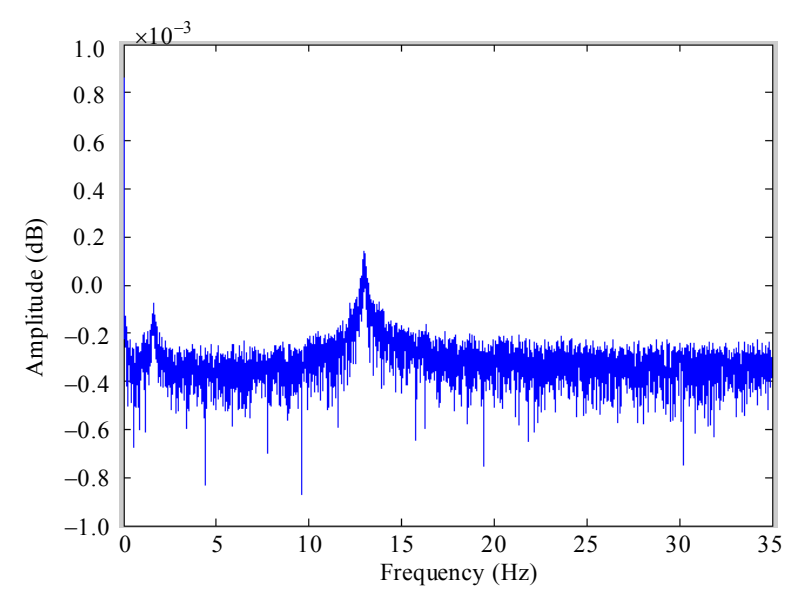

Fig. 8 Vibration frequency is $14 \mathrm{~Hz}$.

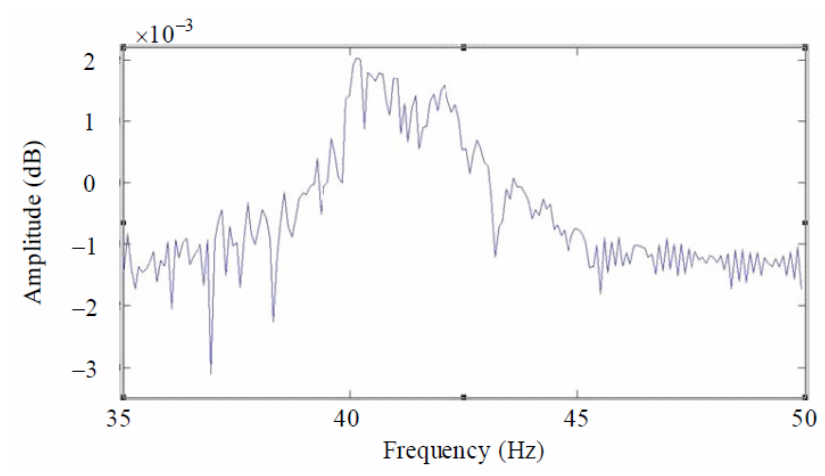

Fig. 9 Vibration frequency is $42 \mathrm{~Hz}$.

The peak value of the frequency domain image where the vibration frequency is about $34 \mathrm{~Hz}$ is not exactly the same as the setting frequency of the vibration table where the vibration frequency is about $32 \mathrm{~Hz}$. The error is shown in Fig. 10.

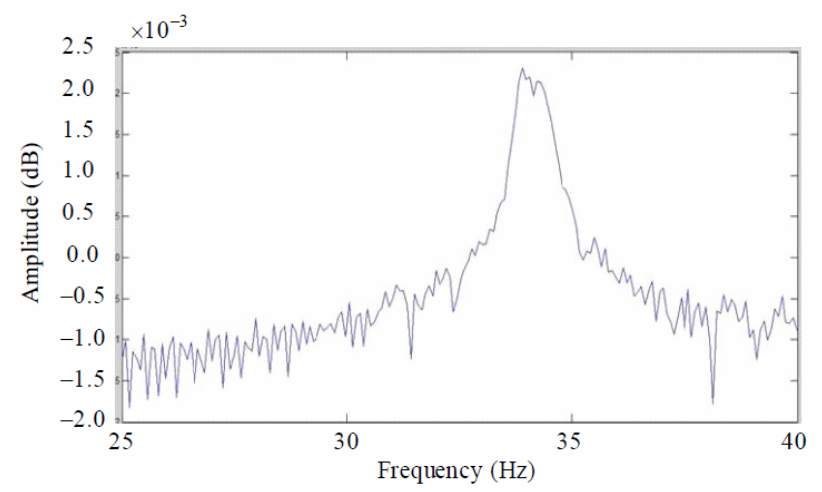

Fig. 10 Vibration frequency is $32 \mathrm{~Hz}$.

Through the measurement of the above experiments, we can see that when the vibration of the vibration source is more intense, the greater the change of the central wavelength is, the greater the vibration frequency is, so this feature can be used in the position around the layout of a series of sensors, analysis of human intrusion, vehicle intrusion, and the waveform of the earth by analyzing the frequency, amplitude, and other parameters of the measured waveform. Furthermore, using the advantages of fiber-optic grating wavelength coding, sensors at different positions are numbered, and when the waveform of the corresponding wavelength changes, the positioning of the entry point can be achieved according to the sensor distribution position.

\section{Conclusions}

This paper mainly introduces the fiber grating vibration sensor used to monitor the degree and orientation of the critical parts and other parts of the attack. Three kinds of structures are tentatively envisioned. The vibration cloud image and the first order natural vibration frequency are obtained by simulation using ANSYS software. This paper determines the strength of the cantilever beam structure and single-beam structure to optimize the design. The data are analyzed by the MATLAB test program. The frequency domain map is used to verify the rationality of the simulation structure.

\section{Acknowledgment}

The authors are grateful to all of the subjects who participated in this research.

Open Access This article is distributed under the terms of the Creative Commons Attribution 4.0 International License (http://creativecommons.org/ licenses/by/4.0/), which permits unrestricted use, distribution, and reproduction in any medium, provided you give appropriate credit to the original author(s) and the source, provide a link to the Creative Commons license, and indicate if changes were made.

\section{References}

[1] C. Wu, Y. Zhang, and B. O. Guan, "Simultaneous measurement of temperature and hydrostatic pressure using Bragg gratings in standard and grapefruit 
microstructured fibers," IEEE Sensors Journal, 2011, 11(2): 489-492.

[2] L. Gao, T. Zhu, M. Deng, K. S. Chiang, X. K. Sun, X. P. Dong, et al., "Long-period fiber grating within d-shaped fiber using magnetic fluid for magnetic-field detection," IEEE Photonics Journal, 2013, 4(6): 2095-2104.

[3] L. Su and S. R. Elliott, "All-fiber microcantilever sensor monitored by a low-cost fiber-to-tip structure with subnanometer resolution," Optics Letters, 2010, 35(8): 1212-1214.

[4] P. Lu, Y. P. Xu, F. Baset, X. Y. Bao, and R. Bhardwaj, "In-line fiber microcantilever vibration sensor," Applied Physics Letters, 2013, 103(21): 211113-1-211113-5.

[5] T. Guo, A. Ivanov, C. K. Chen, and J. Albert, "Temperature-independent tilted fiber grating vibration sensor based on cladding-core recoupling," Optics Letters, 2008, 33(9): 1004-1006.

[6]F. Peng, J. Yang, B. Wu, Y. G. Yuan, X. L. Li, A. Zhou, et al., "Compact fiber optic accelerometer," Chinese Optics Letters, 2012, 10(1): 35-37.
[7]F. Peng, J. Yang, X. L. Li, Y. G. Yuan, B. Wu, A. Zhou, et al., "In-fiber integrated accelerometer," Optics Letters, 2011, 36(11): 2056-2058.

[8] Q. Zhang, T. Zhu, Y. S. Hou, and K. S. Chiang, "All-fiber vibration sensor based on a Fabry-Perot interferometer and a microstructure beam," Journal of the Optical Society of America B-Optical Physics, 2013, 30(5): 1211-1215.

[9] Q. Lin, L. H. Chen, S. Li, and X. Wu. "A high-resolution fiber optic accelerometer based on intracavity phase-generated carrier (PGC) modulation," Measurement Science and Technology, 2011, 22(22): 015303.

[10] T. Guo, L. B. Shang, Y. Ran, B. O. Guan, and J. Albert, "Fiber-optic vector vibroscope," Optics Letters, 2012, 37(13): 2703-2705.

[11] Q. Zhang, T. Zhu, F. Yin, and K. S. Chiang, "Temperature-insensitive real-time inclinometer based on an etched fiber Bragg grating," IEEE Photonics Technology Letters, 2014, 26(10): 1049-1051. 